\title{
Early effects of recollection memory on viewing behavior during visual associative recognition
}

\author{
Daniel R. O'Young \\ Northwestern University \\ Chicago, IL, USA \\ Joel L. Voss \\ Northwestern University \\ Chicago, IL, USA
}

\begin{abstract}
Memory for the context in which items were previously encountered is necessary for everyday tasks. Recollection of item-context associations can facilitate search and support earlier viewing of recognition targets. We used fixation-by-fixation analysis of viewing behavior to characterize visual fixations influenced by recollection during associative recognition. We computed the (1) aggregate proportion of viewing time on target and (2) saccades within target per fixation during recognition testing to measure the influence of recollection on viewing behavior. Proportion of viewing time on target and number of saccades within target increased after the initial fixation of recognition test. These results indicate that recollection effects occurred immediately after the initial fixation of visual associative recognition and that early, prolonged fixations and saccades within selected faces could represent a precise behavioral correlate of recollection memory.
\end{abstract}

Keywords: Eye movement, eye tracking, saccades, region of interest, gaze, memory, recollection, recognition, recall

\section{Introduction}

Memory provides knowledge of past experiences, which can inform behavioral decisions during everyday tasks (Tulving, 1972; Eichenbaum \& Cohen, 2004; Voss $\&$ Paller, 2006). For instance, when meeting a person at a crowded venue, memory for the person's face from previous experiences (i.e. episodic contexts) associated with them, can greatly facilitate recognition-based search. Conscious retrieval of such information from memory, or recollection, seems to provide the greatest benefits to recognition-based search. Although such recollection and search are prevalent in daily behavior, the nature of these

Citation: O’Young, D. R. \& Voss, J. L. (20XX). Early effects of recollection memory on viewing behavior during visual associative recognition.

This article is licensed under a Creative Commons Attribution 4.0 International license. cognitive functions, and the relationships between them, remains a topic of research.

Cognitive processes are known to guide eye-movements towards behaviorally-relevant visual information based on task demands (Buswell, 1935; Yarbus, 1967; Loftus \& Mackworth, 1978; Brockmole \& Henderson, 2006; Henderson et al., 2007; Castelhano et al., 2009). Eye-movement tracking during visual recognition tasks allows precise observation of the extent to which viewing behaviors are influenced by memory (Althoff \& Cohen, 1999). Studies continue to show how recollection memory facilitates recognition-based search while distractors are present, as observed through early increases to viewing time and number of fixations on recognition targets (Ryan et al., 2007, Hannula et al., 2007, 2009; Urgolites et al., 2018). These effects seem to reflect conscious recollection of recognition targets (Chua et al., 2012, 2015; Ramey et al., 2019, Nagy and Kiraly, 2018). Early effects of recollection memory on viewing behavior, within $500-1000 \mathrm{~ms}$ 
of search onset, seem to occur regardless of response demands and even when tasks involve concealing recollection memory (Schwedes \& Wentura, 2012, 2016; Mahoney et al., 2018). Overall, these studies indicate that memory retrieval has characteristic effects on eye-movements during visual search and recognition among distractors.

Other studies have shown that recognition involving conscious recollection occurs within two fixations on faces (Hsiao \& Cotrell 2008; Schwedes \& Wentura, 2011, 2016, 2019) and scenes (Foulsham \& Kingstone, 2012). Schwedes and Wentura (2019) showed that a single fixation was sufficient for above-chance recognition performance, which typically involved recognition based on a sense of familiarity, while a second fixation supported maximal recognition performance, which typically involved recognition based on a sense of recollection. This suggests that this eye-movement pattern provides visual information necessary for face recognition involving recollection memory, which could represent a precise behavioral correlate for this cognitive process.

Our study seeks to further characterize how memory retrieval affects specific fixations during visual search and recognition among distractors. We observed fixations during a task first described by Hannula et al. (2007, 2009), in which subjects studied face-scene pairs then had to select the face that had been studied with given scene cue from an array of faces. We calculated proportion of viewing time on selected faces for each fixation of recognitionbased visual search to determine which fixations contribute to recollection-related effects. We also calculated the number of saccades within target for each of these fixations to determine whether this eye-movement pattern (i.e. target fixations followed by a saccade to another fixation within target) contributed to recollection-related effects. We compared these measures across trials with correct vs. incorrect responses to observe general recollection-related effects. To determine whether these effects were related to recollection memory, half of the subjects also reported whether they could recall the face associated with a given scene cue prior to recognition test - allowing the comparison of trials with correct recognition with vs. without such recall. In contrast to previous research with this task, we performed fixation-by-fixation analysis to determine which fixations - in terms of the order they appear during recognition-based search - show effects of recollection memory. We hypothesize that memory-related effects occur at specific fixations during early recognition-based visual search, which involve prolonged fixations on targets and saccades within targets. We further hypothesize that early effects rely upon recall of search targets. Testing these hypotheses would identify specific eye-movements affected by recollection memory during recognitionguided visual search and provide a precise behavioral correlate for observing such memory.

\section{Methods}

Eye-movements were observed during a task requiring visual search and recognition, based on memory for facescene pairs (Hannula et al., 2009). This task consisted of a study phase where subjects observed a series of face-scene pairs followed by a test phase where subjects selected the face associated with the presented scene from an array including two distractor faces. Subjects indicated their selection with a keyboard button press. To identify recall-related eye-movements, half of the subjects also reported whether they could recall the face associated with each scene during the test phase, which is referred to as the "recall-reporting" group in contrast with the "non-reporting" group.

\section{Participants}

28 healthy adult subjects participated in our study between the ages of 18 and 35 with an average age of $25 \pm 3$ years. Subjects were selected from respondents to recruitment advertising. Each subject provided informed consent to participate in accordance with the Northwestern University Institutional Review Board and received financial compensation for participation.

Half of the subjects were assigned to the recall-reporting group and the other half to the non-reporting group, with 14 subjects in each group.

\section{Procedure}

Each experimental session began with task instructions and eye-tracker setup. Subjects then performed a brief practice version of the recognition task. Subjects were required to respond accurately in this practice version before proceeding to the main experimental task.

Each task block consisted of a study phase followed by a test phase. Each study trial began with a fixation cross for 0.5 seconds, then scene presentation for 1 second, then 
the presentation of a face over the center of the scene for 2 seconds. Study trials were separated by inter-trial intervals between 1 and 5 seconds. Each test phase began after 1min break following the study phase. Each test trial began with a fixation cross for 0.5 seconds, then scene presentation for 1 second, a delay period of $4-8$ seconds, the presentation of three faces for 2 seconds. The associated face was pseudorandomly presented in one of three face locations used across all trials, on the top-left, top-right, and bottomcenter of the screen. Each test trial concluded with a recognition response period, where subjects selected the location of the associated face. Subjects in the recall-reporting group additionally indicated whether they could recall the face associated with each scene during the delay period. Test trials were separated by inter-trial intervals between 8 and 12 seconds.

Declarative responses were reported via button press. A keyboard was positioned in front of the subject with their right hand on the arrow keys. Responses were made with the left, down, and right arrow keys. Recall reporting responses, indicating whether subjects could or could not recall the associated face, were made with the left and right arrow keys, respectively. Recognition responses indicating the location of the associated face were made with the left, right, and down arrow keys (i.e. left for upper left, right for upper right, and down for the lower center). Example trials from the study and test phase are illustrated in Figure 1.

Figure 1: Example trials from study phase and test phase

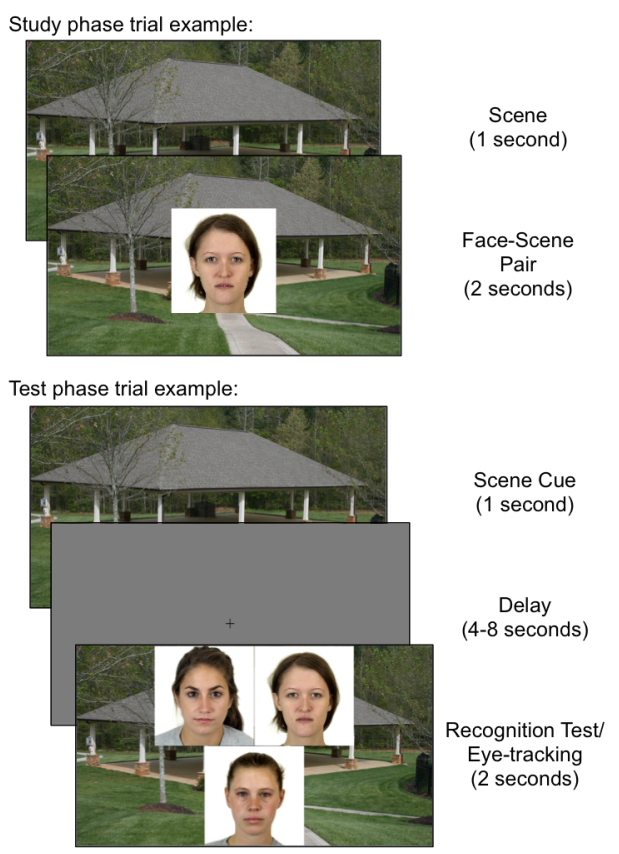

Subjects performed 3 task blocks, each involving 60 study phase trials and 20 test phase trials, except for 4 subjects in the recall-reporting group who performed 4 task blocks, each involving 48 study phase trials and 16 test phase trials.

\section{Materials}

Stimuli included high-resolution, color photographs (at least $1280 \times 720$ pixels). Scene stimuli were selected from the SUN database (Xiao et al., 2016), with each stimulus depicting a unique scene. Scenes were presented across the full screen, approximately $45^{\circ}$ horizontal and $24^{\circ}$ vertical visual angles. Face stimuli were selected from the Chicago Face Database (Ma et al., 2015). Face images were cropped to remove excess background area. Each face image covered $11^{\circ} \times 11^{\circ}$ visual angle. Stimuli were presented on a 23-inch, high-definition computer monitor (1920 x 1080 pixels).

An EyeLink1000 eye-tracking system (SR Research, Ottawa, Canada) was used to record fixation locations at $500 \mathrm{~Hz}$ from the right eye of each subject. Subjects were seated in a comfortable position with their eyes approximately 60 centimeters from the center of the upper half of the computer screen. The tracker was calibrated at the beginning of each experimental block by recording eye positions for five known on-screen reference points, positioned in the middle of the screen and at the center of the top, bottom, left, and right edges. The coordinate location, onset, and duration of each fixation were extracted from the continuous record of eye-position values using standard definitions of fixations, saccades, and blinks. Motion $\left(0.15^{\circ}\right)$, velocity $(30 \%)$, and acceleration $\left(8000^{\circ} / \mathrm{s} 2\right)$ thresholds were used to identify saccades. Events in which the pupil size was very small were classified as blinks. Otherwise, eye movement events falling below the saccade detection thresholds were categorized as fixation events. The average gaze position was calculated over the duration of each fixation event. This system and recording parameters were configured according to recommendations in the EyeLink1000 manual (SR Research 2010).

\section{Design and Analysis}

This experiment was primarily designed to compare viewing behavior directed towards correctly selected targets vs. incorrectly selected distractors. Thus, we performed within-subjects comparisons of the proportion of viewing time and number of saccades within selected faces 
on correct vs. incorrect trials. For recall-reporting subjects, we also compared correct trials with recall vs. correct trials without recall. Lastly, between group comparison of correct trials from recall-reporting vs. non-reporting subjects were used to identify effects related to the additional response demand.

Region-of-interest (ROI) based analysis was used to determine whether fixations were directed the selected face on each test trial. Each selected face ROI consisted of a $5^{\circ} \times 5^{\circ}$ square region centered on the face in each image. Analyses excluded trials where no responses were provided or when visual search began more than $1^{\circ}$ from central fixation, i.e. when faces were presented at recognition test.

To confirm that this task resulted in memory-related effects on viewing time, as described in previous studies, we began by comparing the proportion of viewing time within the selected face ROI on correct vs. incorrect trials across $250 \mathrm{~ms}$ time bins (i.e. 0-250 ms, 250-500 ms, 500$750 \mathrm{~ms}$, etc.) as in previous research (Hannula et al. 2007; Mahoney et al., 2018).

Fixations were then sorted by the order they appeared during visual search for fixation-by-fixation analysis. We calculated the proportion of total viewing time and number of saccades within the selected face ROI by the end of each fixation. The average of these measures was calculated by fixation order for correct trials and incorrect trials. These averages were also calculated for recall-reporting subject for correct trials with and without recall. Fixation-by-fixation analyses were performed on the average number of fixations observed across all trials and subjects.

T-tests were used for within-subject and betweengroup comparisons with statistical significance defined as $\alpha=.05$. MATLAB (2018a) was used to calculate dependent measures for each condition within subjects and perform statistical tests.

\section{Results}

\section{Correct vs. Incorrect Trials}

Correct recognition responses were observed on $73 \pm 2 \%$ of trials. Proportion of viewing time on selected faces increased significantly for correct vs. incorrect trials beginning at the $250-500 \mathrm{~ms}$ time bin $(T(27)=4.21$, $p<.001)$. This effect persisted through subsequent time bins, as summarized in Table 1 and illustrated in Figure 2.

Table 1: Proportion of viewing time on target for correct vs. incorrect trials

\begin{tabular}{c|c|c|ccc}
$\begin{array}{c}\text { Proportion of } \\
\begin{array}{c}\text { Viewing Time } \\
\text { on Target } \\
\text { (by time bin) }\end{array}\end{array}$ & $\begin{array}{c}\text { Correct } \\
\text { Trials } \\
(\text { mean } \pm S E)\end{array}$ & $\begin{array}{c}\text { Incorrect } \\
\text { Trials } \\
(\text { mean } \pm S E)\end{array}$ & $d f$ & $T$ & $p$ \\
\hline $0-250 \mathrm{~ms}$ & $.03 \pm .00$ & $.03 \pm .01$ & 27 & 1.02 & .32 \\
$250-500 \mathrm{~ms}$ & $.33 \pm .01$ & $.23 \pm .02$ & 27 & 4.21 & $<.001^{*}$ \\
$500-750 \mathrm{~ms}$ & $.44 \pm .02$ & $.29 \pm .02$ & 27 & 5.04 & $<.001^{*}$ \\
$750-1000 \mathrm{~ms}$ & $.44 \pm .02$ & $.31 \pm .03$ & 27 & 4.57 & $<.001^{*}$ \\
$1000-1250 \mathrm{~ms}$ & $.45 \pm .01$ & $.28 \pm .02$ & 27 & 5.29 & $<.001^{*}$ \\
$1250-1500 \mathrm{~ms}$ & $.47 \pm .02$ & $.25 \pm .02$ & 27 & 7.17 & $<.001^{*}$ \\
$1500-1750 \mathrm{~ms}$ & $.53 \pm .02$ & $.21 \pm .02$ & 27 & 9.91 & $<.001^{*}$ \\
$1750-2000 \mathrm{~ms}$ & $.56 \pm .02$ & $.22 \pm .02$ & 27 & 11.00 & $<.001^{*}$ \\
\hline
\end{tabular}

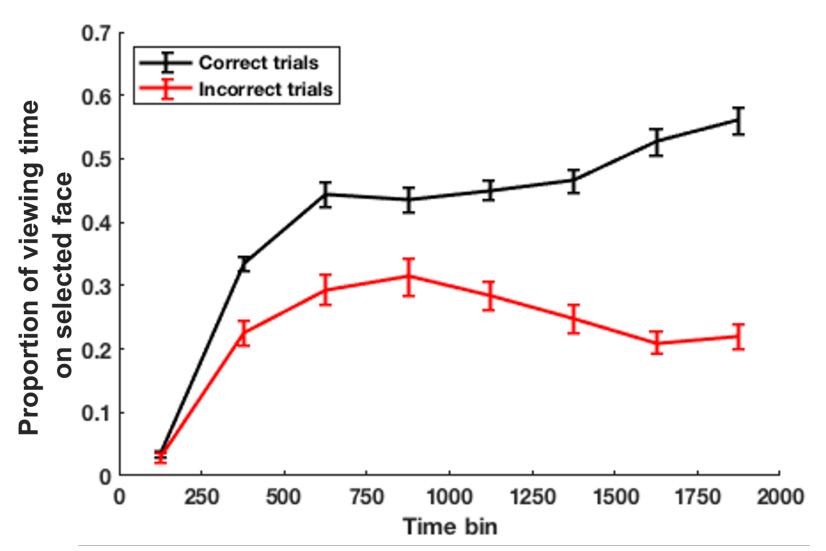

Figure 2: Proportion of viewing time on selected faces for correct vs. incorrect trials

The average number of fixations across all trials for each subject was 6.65土.18. Thus, fixation-by-fixation analyses were performed on the first 6 fixations of recognition test.

Fixation-by-fixation analysis of correct vs. incorrect trials showed that proportion of viewing time increased significantly by the $3^{\text {rd }}$ fixation of recognition-based visual search and that the number of saccades within selected faces increased significantly by the $4^{\text {th }}$ fixation. Proportion of viewing time on selected faces increased to $0.42 \pm .01$ by the $3^{\text {rd }}$ fixation on correct trials, compared to $0.35 \pm .01$ on incorrect trials $(T(27)=5.89, p<0.001)$, while the average number of saccades within selected faces increased to $0.59 \pm .04$ by the $4^{\text {th }}$ fixation on correct trials, compared to $0.45 \pm .04$ on incorrect trials $(T(27)=3.74, p=0.001)$. These increases to proportion of viewing time and number of 
saccades within selected faces persisted through subsequent fixations, as described in Table 2. These results are illustrated in Figure 3.

Table 2: Fixation-by-fixation results for correct vs. incorrect trials

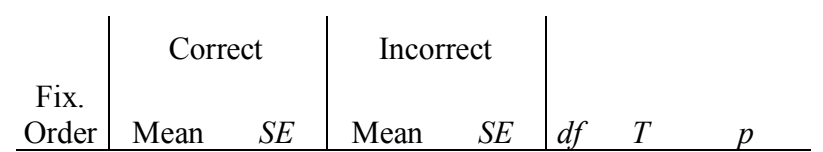

\begin{tabular}{llllllll} 
Order & Mean & $S E$ & Mean & $S E$ & $d f$ & $T$ & $p$ \\
\hline
\end{tabular}

Proportion of viewing time on selected face

\begin{tabular}{l|cc|cc|ccc}
\hline 1 & 0.29 & 0.02 & 0.30 & 0.02 & 27 & -0.40 & 0.69 \\
2 & 0.39 & 0.01 & 0.36 & 0.01 & 27 & 0.88 & 0.39 \\
3 & 0.42 & 0.01 & 0.35 & 0.01 & 27 & 5.89 & $<0.001^{*}$ \\
4 & 0.44 & 0.01 & 0.34 & 0.01 & 27 & 9.24 & $<0.001^{*}$ \\
5 & 0.46 & 0.01 & 0.35 & 0.01 & 27 & 9.72 & $<0.001^{*}$ \\
6 & 0.46 & 0.01 & 0.35 & 0.01 & 27 & 8.54 & $<0.001^{*}$ \\
\hline
\end{tabular}

\begin{tabular}{l|cc|cc|ccc}
\hline \multicolumn{8}{c}{ Saccades within selected face } \\
\hline 2 & 0.22 & 0.02 & 0.22 & 0.02 & 27 & -0.05 & 0.96 \\
3 & 0.39 & 0.03 & 0.35 & 0.03 & 27 & 1.66 & 0.11 \\
4 & 0.59 & 0.04 & 0.45 & 0.04 & 27 & 3.74 & $0.001^{*}$ \\
5 & 0.80 & 0.04 & 0.61 & 0.04 & 27 & 3.63 & $0.001^{*}$ \\
6 & 1.01 & 0.05 & 0.76 & 0.05 & 27 & 4.01 & $<0.001^{*}$ \\
\hline
\end{tabular}
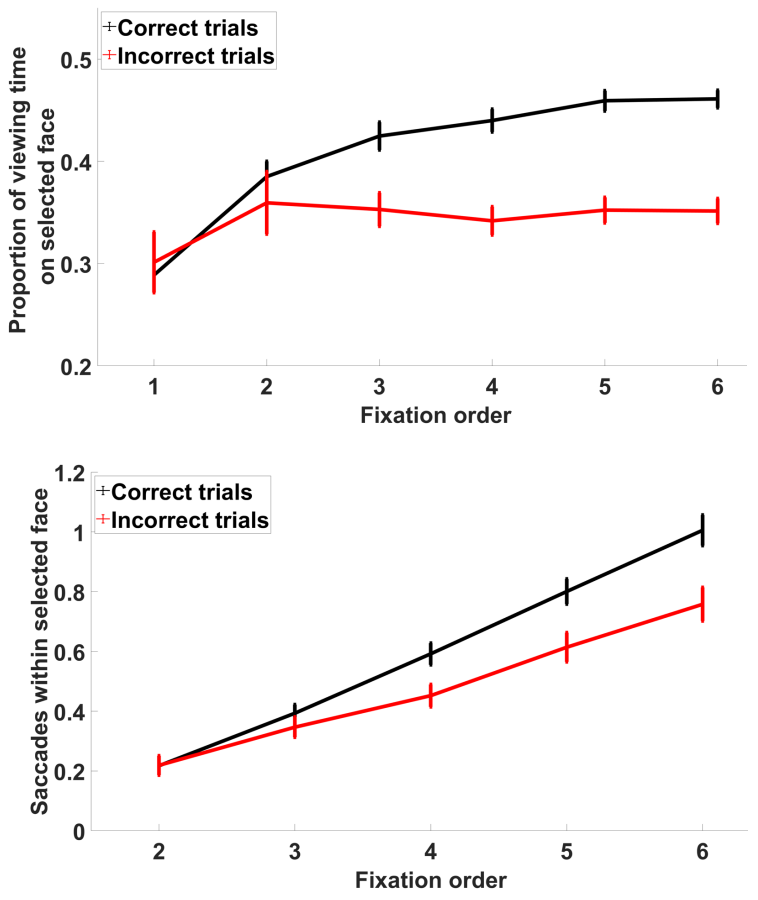

Figure 3: Proportion of viewing time on selected faces (top panel) and average number of saccades within selected faces (bottom panel) for correct vs. incorrect trials

\section{Correct Trials With Recall vs. Without Recall}

For recall-reporting subjects, correct recognition with recall occurred on $46 \pm 4 \%$ of trials, while correct recognition without recall occurred on $26 \pm 4 \%$.

Proportion of viewing time on selected faces increased significantly for correct trials with recall vs. correct trials without recall at the $500-750 \mathrm{~ms}$ time bin $(T(13)=3.86$, $p=.002)$. A trending effect was also observed at the 250$500 \mathrm{~ms}$ time bin $(T(13)=1.83, p=0.09)$. No other significant effects were observed at any other times bins. These results are described in Table 4 and illustrated in Figure 4.

Table 4: Proportion of viewing time on target for correct trials with recall vs. without recall

\begin{tabular}{|c|c|c|c|c|c|}
\hline $\begin{array}{c}\text { Proportion of } \\
\text { Viewing Time } \\
\text { on Target } \\
\text { (by time bin) }\end{array}$ & \begin{tabular}{|c} 
Correct \\
Trials \\
With \\
Recall \\
$($ mean $\pm S E)$
\end{tabular} & $\begin{array}{c}\text { Correct } \\
\text { Trials } \\
\text { Without } \\
\text { Recall } \\
(\text { mean } \pm S E)\end{array}$ & $d f$ & $T$ & $p$ \\
\hline $0-25$ & $.04 \pm$ & $.03=$ & 13 & 1.10 & .29 \\
\hline $250-500 \mathrm{~ms}$ & $.38 \pm$ & $.29 \pm .05$ & 13 & 1.83 & .09 \\
\hline $500-750 \mathrm{~ms}$ & $.49 \pm .04$ & $.32 \pm .04$ & 13 & 3.86 & $.002 *$ \\
\hline $750-1000 \mathrm{~ms}$ & $.45 \pm .04$ & $.41 \pm .04$ & 13 & .95 & .36 \\
\hline $1000-1250 \mathrm{~ms}$ & $.44 \pm .04$ & $.39 \pm .04$ & 13 & 1.13 & .28 \\
\hline $1250-1500 \mathrm{~ms}$ & $.47 \pm$ & $.44 \pm .04$ & 13 & .56 & .58 \\
\hline $1500-1750 \mathrm{~ms}$ & $.57 \pm .04$ & $.52 \pm .03$ & 13 & 1.51 & .15 \\
\hline $1750-2000 \mathrm{~ms}$ & $.62 \pm .04$ & $.58 \pm .05$ & 13 & .83 & .42 \\
\hline
\end{tabular}

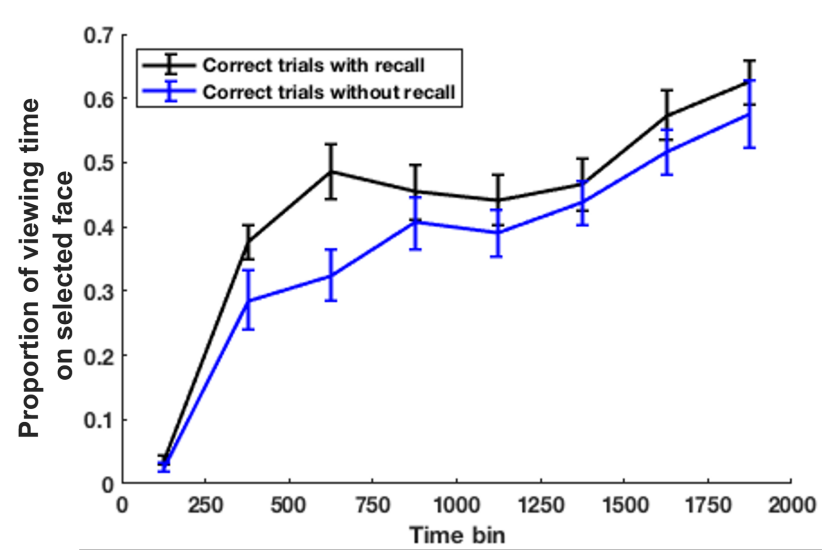

Figure 4: Proportion of viewing time on selected face for correct trials with recall vs. correct trials without recall

Fixation-by-fixation analysis of correct trials with vs. without recall showed that proportion of viewing time increased significantly during the $2^{\text {rd }}, 3^{\text {rd }}$, and $4^{\text {th }}$ fixations of recognition-based visual search and that the number of saccades within selected faces increased significantly by 
during the $3^{\text {rd }}$ fixation. Proportion of viewing time on selected faces increased to $0.43 \pm .03$ by the $2^{\text {nd }}$ fixation on correct trials with recall, compared to $0.31 \pm .03$ on correct trials without recall $(T(13)=2.28, p=0.04)$. For the $3^{\text {rd }}$ fixation, proportion of viewing time increased further to $0.46 \pm .03$ on correct trials with recall, compared to $0.34 \pm .03$ on correct trials without recall $(T(13)=3.17$, $p=0.01)$. For the $4^{\text {th }}$ fixation, proportion of viewing time increased to $0.46 \pm .03$ on correct trials with recall, compared to $0.39 \pm .03$ on correct trials without recall $(T(13)=2.23, p=.04)$. The average number of saccades within selected faces increased to $0.42 \pm .06$ by the $3^{\text {rd }}$ fixation on correct trials with recall, compared to $0.24 \pm .06$ on correct trials without recall $(T(13)=2.45, p=0.03)$. These results, for proportion of viewing time and number of saccades within selected are described in Table 5. These results are illustrated in Figure 5.

Table 5: Fixation-by-fixation results for correct trials with vs. without recall

\begin{tabular}{r|cc|cc|ccc} 
& $\begin{array}{c}\text { Correct trials } \\
\text { with } \\
\text { Recall }\end{array}$ & $\begin{array}{c}\text { Correct trials } \\
\text { without } \\
\text { Recall }\end{array}$ & & & & \\
$\begin{array}{c}\text { Fix. } \\
\text { Order }\end{array}$ & Mean & $S E$ & Mean & $S E$ & $d f$ & $T$ & $p$ \\
\hline \multicolumn{7}{|c}{ Proportion of viewing time on selected face } \\
\hline 1 & 0.30 & 0.03 & 0.29 & 0.03 & 13 & 0.23 & 0.82 \\
2 & 0.43 & 0.03 & 0.31 & 0.03 & 13 & 2.28 & $0.04^{*}$ \\
3 & 0.46 & 0.03 & 0.34 & 0.03 & 13 & 3.17 & $0.01^{*}$ \\
4 & 0.46 & 0.03 & 0.39 & 0.03 & 13 & 2.23 & $0.04^{*}$ \\
5 & 0.48 & 0.03 & 0.43 & 0.03 & 13 & 1.68 & 0.12 \\
6 & 0.49 & 0.02 & 0.42 & 0.02 & 13 & 2.43 & 0.03 \\
\hline
\end{tabular}

\begin{tabular}{l|cc|cc|ccc}
\multicolumn{7}{c}{ Saccades within selected face } \\
\hline 2 & 0.22 & 0.03 & 0.18 & 0.03 & 13 & 0.82 & 0.43 \\
3 & 0.42 & 0.06 & 0.24 & 0.06 & 13 & 2.45 & $0.03^{*}$ \\
4 & 0.61 & 0.08 & 0.44 & 0.08 & 13 & 1.77 & 0.10 \\
5 & 0.80 & 0.09 & 0.67 & 0.09 & 13 & 1.24 & 0.24 \\
6 & 1.00 & 0.10 & 0.90 & 0.10 & 13 & 0.87 & 0.40 \\
\hline
\end{tabular}
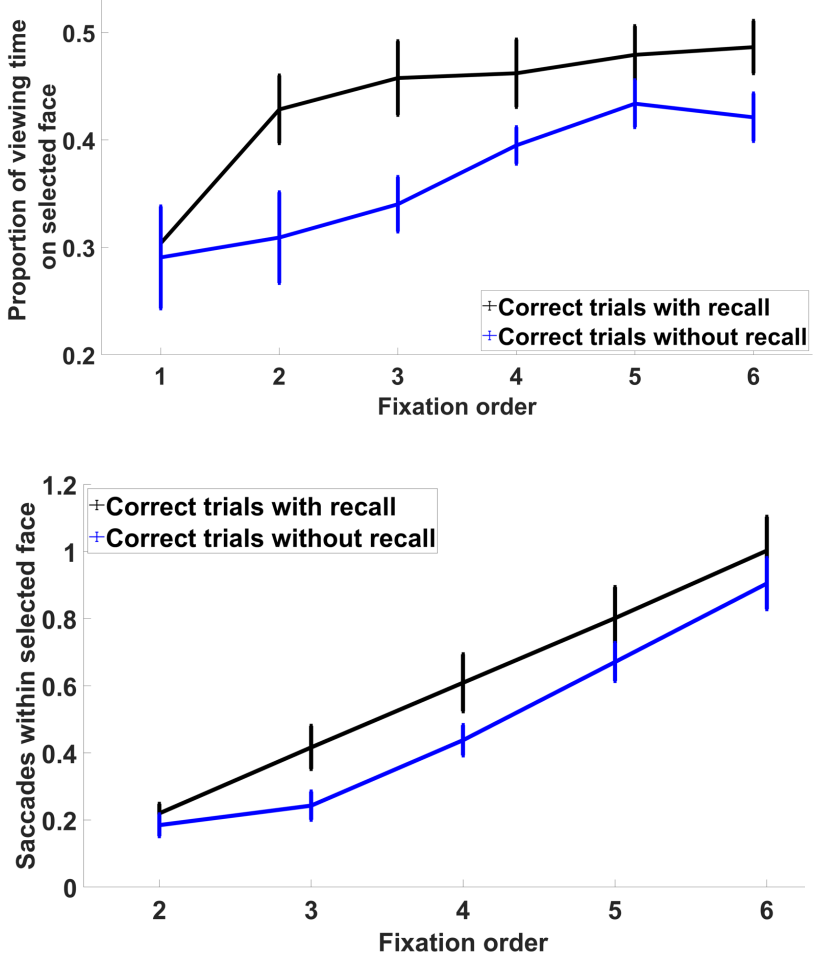

Figure 5: Proportion of viewing time on selected faces (top panel) and average number of saccades within selected faces (bottom panel) for correct trials with vs. without recall

\section{Recall-Reporting vs. Non-Reporting Group}

Recognition performance did not differ significantly between the recall-reporting and non-reporting groups, with correct responses observed on $72 \pm 3 \%$ of trials in the recall-reporting group and $73 \pm 4 \%$ in the non-reporting group $(T(26)=-.17, \mathrm{p}=.87)$. Fixation-by-fixation analysis did not show significant effects on proportion of viewing time on selected faces nor average number of saccades within selected faces. These results are summarized in Table 6 and Figure 6 .

Table 6: Fixation-by-fixation results for the recall-reporting group vs. non-reporting group

\begin{tabular}{c|cc|cc|ccc} 
Fix. & $\begin{array}{c}\text { Correct trials } \\
\text { Recall-report- } \\
\text { ing group }\end{array}$ & $\begin{array}{c}\text { Correct trials } \\
\text { Non-reporting } \\
\text { group }\end{array}$ & & & & \\
Order & Mean $\quad S E$ & Mean & $S E$ & $d f$ & $T$ & $p$ \\
\hline \multicolumn{7}{c}{ Proportion of viewing time on selected face } \\
\hline 1 & 0.29 & 0.02 & 0.29 & 0.02 & 26 & 0.12 & 0.90
\end{tabular}




\begin{tabular}{l|ll|ll|lll}
2 & 0.37 & 0.02 & 0.40 & 0.02 & 26 & -0.82 & 0.42 \\
3 & 0.40 & 0.02 & 0.45 & 0.02 & 26 & -1.74 & 0.09 \\
4 & 0.43 & 0.02 & 0.45 & 0.02 & 26 & -1.21 & 0.24 \\
5 & 0.46 & 0.02 & 0.46 & 0.02 & 26 & -0.29 & 0.78 \\
6 & 0.46 & 0.01 & 0.46 & 0.01 & 26 & -0.45 & 0.66 \\
\hline \multicolumn{7}{c}{ Saccades within selected face } \\
\hline 2 & 0.20 & 0.02 & 0.23 & 0.02 & 26 & -0.83 & 0.41 \\
3 & 0.34 & 0.04 & 0.44 & 0.04 & 26 & -1.78 & 0.09 \\
4 & 0.53 & 0.04 & 0.65 & 0.04 & 26 & -1.82 & 0.08 \\
5 & 0.74 & 0.05 & 0.86 & 0.05 & 26 & -1.44 & 0.16 \\
6 & 0.96 & 0.06 & 1.05 & 0.06 & 26 & -0.79 & 0.44 \\
\hline
\end{tabular}
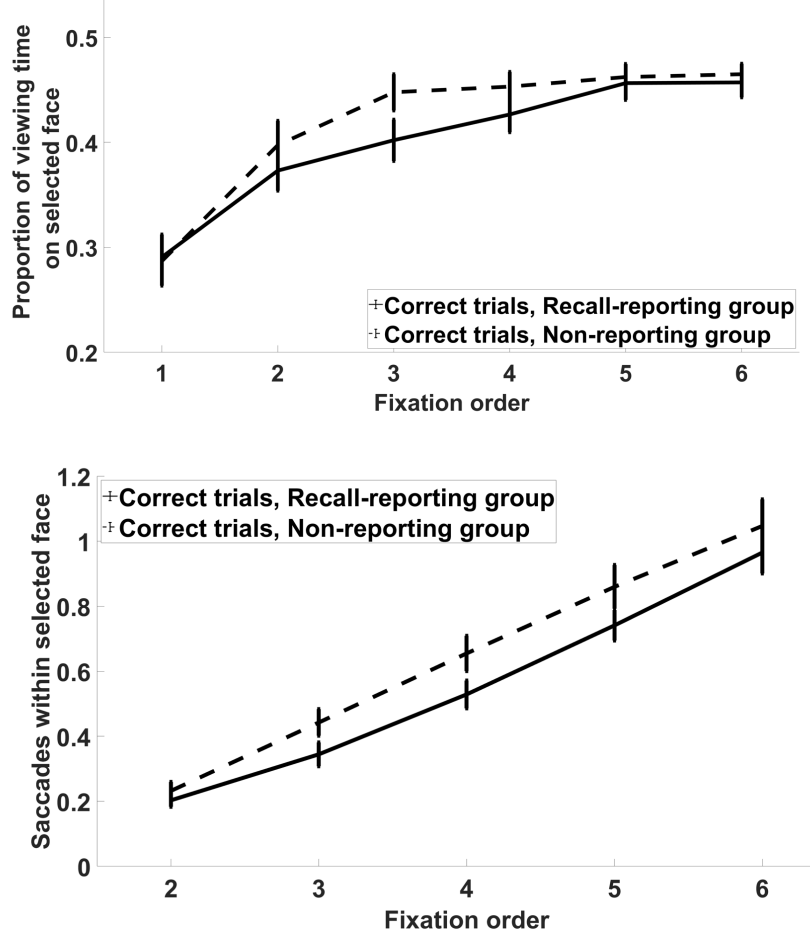

Figure 6: Proportion of viewing time on selected faces (top panel) and average number of saccades within selected faces (bottom panel) for correct trials from the recall-reporting group vs. non-reporting group.

\section{Discussion}

This study tested how recollection memory affects viewing behaviors during visual associative recognition and sought to characterize specific eye-movements that demonstrate these effects. To test the hypothesis that specific fixations were influenced by recollection memory, we performed eye-movement tracking during visual search requiring associative recognition based on recollection of studied scene-face pairs. Fixation-by-fixation analysis showed that recollection effects emerged by the $3^{\text {rd }}$ fixations of visual search, when significant increases to proportion of viewing time and saccades within selected were observed. Earlier effects, by the $2^{\text {rd }}$ fixation, occurred when subjects could recall correctly recognized faces, but not when correct recognition was achieved without such recall. Together, these results show that recollection effects emerge by the $2^{\text {nd }}$ fixation of visual search, which seems to rely upon conscious recall of search targets. These indicates that early, prolonged fixations and saccades within recognized faces corresponds with the behavioral effects of recollection memory.

In contrast to previous research involving this visual associative recognition task that analyzed proportion of viewing time across time bins, our results include fixationby-fixation analyses, which demonstrate how specific fixations - in terms of the order they appear during search are affected by recollection memory. These fixations seem to comprise previously described effects on proportion of viewing time based on time bin analyses. Our results additionally show that conscious recall of search targets supports early effects, during the $500-750 \mathrm{~ms}$ time bin or the $2^{\text {nd }}$ fixation of visual search.

Fixation-by-fixation analyses also involved comparisons of the number of saccades within selected faces, due to previous studies showing how this viewing behavior (i.e. second fixation onto a non-redundant region of a face) seems to support face recognition that involves recollection (Schwedes \& Wentura, 2019). This analysis showed that this behavior also occurred with associative recognition and that earlier expression of this behavior (by the $3^{\text {rd }}$ fixation of visual search) seemed to rely upon conscious recall of search targets.

To determine whether recall-reporting affected proportion of viewing time or saccades within selected faces, we compared these measures on correct trials from the recallreporting group vs. non-reporting group. Significant differences were not observed for either of these measures, although there were trends of decreased proportion of viewing time for the $3^{\text {rd }}$ fixation and saccades within selected faces for the $3^{\text {rd }}$ and $4^{\text {th }}$ fixations for the recall-reporting vs. non-reporting groups. 
Taken together, our results indicate that recollection memory, involving conscious recall of search targets, supports early effects on viewing behaviors during visual associative recognition, which can be observed through prolonged fixations and saccades within recognized targets. These results provide a novel, behavioral perspective for understanding the relationship between recollection and viewing behaviors during visual associative recognition. This informs the interpretation of previous research involving this experimental task. For instance, the fixation patterns observed in our study correspond with increases to proportion of viewing time on target, observed when subjects are able to consciously recall and confidently recognize search targets (Chua et al., 2012, 2015). Other studies have also shown that increased viewing time on target when subjects were not required to make recognition responses (Hannula et al., 2007; Nagy and Kiraly, 2018) and when instructed to "feign memory impairment" (Mahoney et al., 2018). Additionally, the neuroimaging study by Hannula \& Ranganath (2009) showed that this effect was associated with coordinated activation of brain regions thought to support episodic memory retrieval - as in hippocampus and prefrontal cortex.

Our analysis of saccades within faces provides further evidence that the second fixation on non-redundant regions of a face (i.e. fixations resulting from saccades within a face) are critical for recollection memory (Schwedes \& Wentura, 2019). While previous studies demonstrate how this viewing behavior supports recollection during item recognition, we show that this behavior also occurs during associative recognition based on recollection memory. These observations demonstrate that the visual information provided by this viewing behavior supports memory retrieval processes involved in recollection.

The recollection-related effects that we observed suggests that viewing behavior reflects the accumulation of decision-making information over time, which is thought to determine responses and reaction times during recognition-based (Ratcliff \& McKoon, 2008), perceptual (Heekeren et al., 2004; Philiastades et al., 2005, 2006, 2007; Siegel et al., 2011; Kelly \& O'Connell, 2013), and valuebased decisions (Hunt et al. 2012; Krajbich et al., 2010, 2011). Krajbich et al. $(2010,2011)$ showed that this information accumulation process can be observed through eye-movement tracking during decisions involving the selection visual objects. They showed that fixation times could be used as a measure of information accumulation that corresponds with selection of the fixated object. It is feasible that fixation times could reflect this decision-making process during associative recognition and other recollection-based decisions. Although our results also show relationships between fixation time and selection responses, testing the relationship between fixation times and information accumulation requires responses during visual search - unlike the present experiment where responses were made after the visual search period. However, our results characterize how specific fixations seem to provide information for decisions based on recollection and associative recognition. These observations provide motivation for future research into computational models that relate associative memory retrieval and recollection to viewing behaviors involved in decision-making processes.

Overall, our results further characterize the relationship between recollection memory and viewing behaviors involved in associative recognition of faces. We show that recollection affects early viewing behaviors, which involve prolonged fixations and saccades within recognized faces. These results further demonstrate that eye-movement tracking allows precise observation of how memory influences behavior and when these effects occur.

\section{Conclusion}

This study sought to characterize effects of recollection memory on viewing behaviors involved in visual associative recognition targets among familiar distractors. Eyemovement tracking during visual associative recognition of faces showed that recollection results in early increases to viewing time on targets, which involves prolonged fixations and saccades within target. We used fixation-by-fixation analyses to show that these effects on viewing behavior emerged immediately after the initial fixation of viewing. This study is the first to characterize specific viewing behaviors affected by recollection memory and when they occur during visual associative recognition, which seems to represent a precise, behavioral correlate for observing recollection memory.

\section{Ethics and Conflict of Interest}

The author(s) declare(s) that the contents of the article are in agreement with the ethics described in http://biblio.unibe.ch/portale/elibrary/BOP/jemr/ethics.html and 
that there is no conflict of interest regarding the publication of this paper.

\section{Acknowledgements}

This research was supported in part by grant P50MH094263, T32-MH067564, and R21MH108863 from the National Institute of Mental Health.

The authors thank Drs. Donna J. Bridge, Thorsten Khant, and Ken A. Paller for their guidance and feedback.

\section{References}

Althoff, R. R., \& Cohen, N. J. (1999). Eye-movementbased memory effect: a reprocessing effect in face perception. Journal of Experimental Psychology: Learning, Memory, and Cognition, 25(4), 997.

Brockmole, J. R., \& Henderson, J. M. (2006). Recognition and attention guidance during contextual cueing in real-world scenes: Evidence from eye movements. The Quarterly journal of experimental psychology, 59(7), 1177-1187.

Buswell, G. T. (1935). How people look at pictures: a study of the psychology and perception in art.

Castelhano, M. S., Mack, M. L., \& Henderson, J. M. (2009). Viewing task influences eye movement control during active scene perception. Journal of vision, 9(3), 6-6.

Chua, E. F., Hannula, D. E., \& Ranganath, C. (2012). Distinguishing highly confident accurate and inaccurate memory: Insights about relevant and irrelevant influences on memory confidence. Memory, 20(1), 48-62.

Chua, E. F., \& Solinger, L. A. (2015). Building metamemorial knowledge over time: insights from eye tracking about the bases of feeling-of-knowing and confidence judgments. Frontiers in psychology, 6, 1206.

Chun, M. M., \& Jiang, Y. (1998). Contextual cueing: Implicit learning and memory of visual context guides spatial attention. Cognitive psychology, 36(1), 28-71.

Eichenbaum, H., \& Cohen, N. J. (2004). From conditioning to conscious recollection: Memory systems of the brain (No. 35). Oxford University Press on Demand.

Hannula, D. E., Ryan, J. D., Tranel, D., \& Cohen, N. J. (2007). Rapid onset relational memory effects are evident in eye movement behavior, but not in hippocampal amnesia. Journal of cognitive neuroscience, 19(10), 1690-1705.

Hannula, D. E., Ryan, J. D., Tranel, D., \& Cohen, N. J. (2007). Rapid onset relational memory effects are evident in eye movement behavior, but not in hippocampal amnesia. Journal of cognitive neuroscience, 19(10), 1690-1705.

Hannula, D. E., \& Ranganath, C. (2009). The eyes have it: hippocampal activity predicts expression of memory in eye movements. Neuron, 63(5), 592-599. 
Hannula, D. E., Althoff, R. R., Warren, D. E., Riggs, L., Cohen, N. J., \& Ryan, J. D. (2010). Worth a glance: using eye movements to investigate the cognitive neuroscience of memory. Frontiers in human neuroscience, 4, 166.

Henderson, J. M., Brockmole, J. R., Castelhano, M. S., \& Mack, M. (2007). Visual saliency does not account for eye movements during visual search in real-world scenes. In Eye movements (pp. 537-III). Elsevier.

Heekeren, H. R., Marrett, S., Bandettini, P. A., \& Ungerleider, L. G. (2004). A general mechanism for perceptual decision-making in the human brain. Nature, 431(7010), 859.

Hsiao, J. H. W., \& Cottrell, G. (2008). Two fixations suffice in face recognition. Psychological Science, 19(10), 998-1006.

Hunt, L. T., Kolling, N., Soltani, A., Woolrich, M. W., Rushworth, M. F., \& Behrens, T. E. (2012). Mechanisms underlying cortical activity during value-guided choice. Nature neuroscience, 15(3), 470.

Kelly, S. P., \& O'Connell, R. G. (2013). Internal and external influences on the rate of sensory evidence accumulation in the human brain. Journal of Neuroscience, 33(50), 19434-19441.

Krajbich, I., Armel, C., \& Rangel, A. (2010). Visual fixations and the computation and comparison of value in simple choice. Nature neuroscience, 13(10), 1292.

Krajbich, I., \& Rangel, A. (2011). Multialternative driftdiffusion model predicts the relationship between visual fixations and choice in value-based decisions. Proceedings of the National Academy of Sciences, 108(33), 13852-13857.

Loftus, G. R., \& Mackworth, N. H. (1978). Cognitive determinants of fixation location during picture viewing. Journal of Experimental Psychology: Human perception and performance, 4(4), 565.

Ma, D. S., Correll, J., \& Wittenbrink, B. (2015). The Chicago face database: A free stimulus set of faces and norming data. Behavior research methods, 47(4), 1122-1135.

Mahoney, E. J., Kapur, N., Osmon, D. C., \& Hannula, D. E. (2018). Eye tracking as a tool for the detection of simulated memory impairment. Journal of Applied Research in Memory and Cognition, 7(3), 441-453.
Nagy, M., \& Király, I. (2018). Evidence of relational retrieval, even in the absence of the relational eye movement effect (REME). Consciousness and cognition, 66, 40-53.

Philiastides, M. G., \& Sajda, P. (2005). Temporal characterization of the neural correlates of perceptual decision making in the human brain. Cerebral cortex, 16(4), 509-518.

Philiastides, M. G., Ratcliff, R., \& Sajda, P. (2006). Neural representation of task difficulty and decision making during perceptual categorization: a timing diagram. Journal of Neuroscience, 26(35), 8965-8975.

Philiastides, M. G., \& Sajda, P. (2007). EEG-informed fMRI reveals spatiotemporal characteristics of perceptual decision making. Journal of Neuroscience, 27(48), 13082-13091.

Ramey, M. M., Yonelinas, A. P., \& Henderson, J. M. (2019). Conscious and unconscious memory differentially impact attention: Eye movements, visual search, and recognition processes. Cognition, 185, 71-82.

Ratcliff, R. (1978). A theory of memory retrieval. Psychological review, 85(2), 59.

Ratcliff, R., \& McKoon, G. (2008). The diffusion decision model: theory and data for two-choice decision tasks. Neural computation, 20(4), 873-922.

Schwedes, C., \& Wentura, D. (2012). The revealing glance: Eye gaze behavior to concealed information. Memory \& cognition, 40(4), 642-651.

Schwedes, C., \& Wentura, D. (2016). Through the eyes to memory: Fixation durations as an early indirect index of concealed knowledge. Memory \& cognition, 44(8), 1244-1258.

Schwedes, C., \& Wentura, D. (2019). The relevance of the first two eye fixations for recognition memory processes. Memory, 27(6), 792-806.

Siegel, M., Engel, A. K., \& Donner, T. H. (2011). Cortical network dynamics of perceptual decision-making in the human brain. Frontiers in human neuroscience, 5,21 .

SR Research. (2010). EyeLink 1000 User's Manual, Version 1.5. 2.

Tulving, E. (1972). Episodic and semantic memory. Organization of memory, 1, 381-403. 
Urgolites, Z. J., Smith, C. N., \& Squire, L. R. (2018). Eye movements support the link between conscious memory and medial temporal lobe function. Proceedings of the National Academy of Sciences, 115(29), 7599-7604.

van Asselen, M., Sampaio, J., Pina, A., \& CasteloBranco, M. (2011). Object based implicit contextual learning: A study of eye movements. Attention, Perception, \& Psychophysics, 73(2), 297-302.

Voss, J. L., \& Paller, K. A. (2006). Fluent conceptual processing and explicit memory for faces are electrophysiologically distinct. Journal of Neuroscience, 26(3), 926-933.

Xiao, J., Ehinger, K. A., Hays, J., Torralba, A., \& Oliva, A. (2016). Sun database: Exploring a large collection of scene categories. International Journal of Computer Vision, 119(1), 3-22.

Yarbus, A. L. (1967). Eye movements during perception of complex objects. In Eye movements and vision (pp. 171-211). Springer, Boston, MA. 